\title{
Obesity: a new risk factor for cardiac syndrome $\mathrm{X}$ ?
}

\author{
Editorial to: "Obesity, inflammation and brachial artery flow-mediated dilatation: \\ therapeutic targets in patients with microvascular angina (cardiac syndrome $\mathrm{X}$ )" \\ by $P$. Ong et al.
}

\author{
Priscilla Lamendola • Gaetano Antonio Lanza
}

Published online: 29 April 2012

(C) Springer Science+Business Media, LLC 2012

Cardiac X syndrome (CSX) is typically characterized by exercise-induced angina, findings of myocardial ischemia on non invasive stress tests but normal coronary arteries at angiography [1]. Coronary microvascular dysfunction (CMVD) is responsible for CSX in most patients, and may involve an impairment of both endothelium-dependent and endothelium-independent microvascular dilatation, as well as an enhanced microvascular constriction [1].

There is no apparent increase in the risk of major coronary events in CSX, but about $20 \%$ of patients show progressive worsening of symptoms and quality of life, despite optimal medical therapy. The reasons for drug failure in these patients are likely to be multiple, and include an increased painful perception of cardiac stimuli, but also a wide heterogeneity of CMVD mechanisms, which can make it difficult to appropriately target medications in individual patients [2].

Together with a possible role of classical cardiovascular risk factors (CVRFs, i.e., hypertension, smoking, dyslipidemia, diabetes), several other conditions have been suggested to variably contribute to CMVD in CSX, including insulin resistance, inflammation, abnormal adrenergic activity and (in women) estrogen deficiency [1].

In this issue, Ong et al. report some new interesting findings, which contribute to build up the puzzle of the pathogenesis of CSX [3]. The authors assessed brachial flow-mediated dilation (FMD) in 34 CSX patients and in 12 healthy subjects. Compared to controls, patients

P. Lamendola $\cdot$ G. A. Lanza $(\bowtie)$

Istituto di Cardiologia, Università Cattolica del Sacro Cuore,

Largo A. Gemelli, 8,

00168 Roma, Italy

e-mail: g.a.lanza@rm.unicatt.it showed a lower FMD (median [interquartile range] $8.5 \%$ [7.3-9.3] vs. $4.7 \%$ [2.7-7.2], $p<0.01)$, demonstrating systemic endothelial dysfunction (ED). Predictors of ED in CSX patients included hypertension, levels of C-reactive protein (CRP) and obesity (body mass index $\geq 30 \mathrm{~kg} / \mathrm{m}^{2}$ ).

These results confirm previous data that in CSX the vascular abnormality may extend beyond coronary microcirculation and involve ED in peripheral arteries, and also further support the role of inflammation as a pathogenetic factor in these patients. Indeed, CRP levels were previously found increased in CSX patients, and were also shown to correlate with systemic ED and with endotheliumdependent CMVD [4-6]. Notably, in a recent study we found that CRP levels were the only independent predictor of endothelium-independent CMVD in a group of CSX patients [7].

A novel finding in the study by Ong et al. is the association of hypertension and obesity with ED in CSX. Other studies failed to show hypertension as an independent predictor of ED in these patients [7]. Thus this relation deserves further investigation in larger studies. On the other hand, no previous study assessed whether obesity can contribute to ED in CSX, although obesity was associated with ED in other clinical settings [8], and was also associated with coronary ED in a mixed group of patients with normal or mildly diseased coronary arteries [9].

Obesity can induce ED by several mechanisms. It is usually associated with other CVRFs, which can by themselves induce ED, but it often also results in insulin resistance, increased oxidative stress, release of pro-inflammatory and vasoactive substances (e.g., endothelin-1, angiotensin), and activation of the adrenergic system, all of which negatively affect vascular function $[8,9]$. 
Importantly, the observation that obesity can contribute to ED in CSX has potential implications for patient management [3].

The study by Ong et al. leaves opened some relevant questions regarding the role of obesity in CSX.

First, the prevalence of obesity in CSX should be assessed in larger populations of patients to better understand its potential impact in this clinical condition.

A second issue is whether the effects of obesity on FMD in CSX reliably reflect detrimental effects on coronary microvascular function. Indeed, FMD explores peripheral macrovascular endothelial function, whereas CSX symptoms are related to microvascular coronary abnormalities which also involve endothelium-independent mechanisms $[1,9]$.

Whether FMD can be taken as a valid surrogate end-point to predict the effect of treatment on CMVD also remains to be established. In a recent study of young morbidly obese patients without cardiovascular disease, we have indeed shown that weight loss by bariatric surgery significantly improved both peripheral ED and CMVD, but systemic ED improvement only correlated with that of endotheliumdependent CMVD, but not with that of endotheliumindependent CMVD [10].

Finally, the study by Ong et al. [3] was probably underpowered to demonstrate an independent association of obesity with ED. Thus further studies, with larger numbers of patients, are necessary to completely define the role of obesity in CSX patients.

\section{References}

1. Lanza GA, Crea F. Primary coronary microvascular dysfunction: clinical presentation, pathophysiology, and management. Circulation. 2010;121:2317-25.

2. Kaski JC, Valenzuela Garcia LF. Therapeutic options for the management of patients with cardiac syndrome X. Eur Heart J. 2001;22:283-93.

3. Ong P, Sivanathan R, Borgulya G, et al. Obesity, inflammation and brachial artery flow-mediated dilatation: therapeutic targets in patients with microvascular angina (cardiac X syndrome). Cardiovasc Drug Ther. 2012;26: this issue.

4. Tondi R, Santoliquido A, Di Giorgio A, et al. Endothelial dysfunction as assessed by flow-mediated dilation in patients with cardiac syndrome X: role of inflammation. Eur Rev Med Pharmacol Sci. 2011;15:1074-7.

5. Lanza GA, Sestito A, Cammarota G, et al. Assessment of systemic inflammation and infective pathogen burden in patients with cardiac syndrome X. Am J Cardiol. 2004;94:40-4.

6. Teragawa H, Fukuda Y, Matsuda K, et al. Relation between C reactive protein concentrations and coronary microvascular endothelial function. Heart. 2004;90:750-4.

7. Sestito A, Lanza GA, Di Monaco A, et al. Relation between cardiovascular risk factors and coronary microvascular dysfunction in cardiac syndrome X. J Cardiovasc Med. 2011;12:322-7.

8. Caballero AE. Endothelial dysfunction in obesity and insulin resistence: a road to diabetes and heart disease. Obes Res. 2003;11:1278-89.

9. Al Suwaidi J, Higano ST, Holmes DR, et al. Obesity is independently associated with coronary endothelial dysfunction in patients with normal or mildly diseased coronary arteries. J Am Coll Cardiol. 2001;37:1523-8.

10. Nerla R, Tarzia P, Sestito A, et al. Effect of bariatric surgery on peripheral flow-mediated dilation and coronary microvascular function. Nutr Metab Cardiovasc Dis. 2010; Dec 24. [Epub ahead of print] 